\title{
Rehabilitation Training Combined with Jiaji Electroacupuncture Can Promote the Recovery of Muscle Group Function and Improve the Quality of Life in Patients with Upper Limb Peripheral Nerve Injury
}

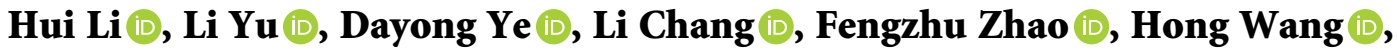 \\ and Tiance Zhang iD \\ Department of Rehabilitation, The First Hospital of Qiqihar, Affiliated Qiqihar Hospital, Southern Medical University, \\ Qiqihar 161000, Heilongjiang, China \\ Correspondence should be addressed to Tiance Zhang; zhangtiance@qhrdyyy.org.cn
}

Received 2 November 2021; Accepted 23 November 2021; Published 20 December 2021

Academic Editor: Kalidoss Rajakani

Copyright (C) 2021 Hui Li et al. This is an open access article distributed under the Creative Commons Attribution License, which permits unrestricted use, distribution, and reproduction in any medium, provided the original work is properly cited.

\begin{abstract}
This study was designed to probe into the improvement of rehabilitation training combined with Jiaji electroacupuncture intervention on patients with upper limb peripheral nerve injury. A total of 114 patients with peripheral nerve injury of upper limbs in our hospital from August 2017 to November 2019 were collected as the research participants. Among them, 59 in the control group (CG) received rehabilitation training alone, while 65 in the observation group (OG) received rehabilitation training combined with Jiaji electroacupuncture intervention. The therapeutic efficacy, Barthel index, and Fugl-Meyer assessment score, motor nerve conduction velocity, sensory nerve conduction velocity and amplitude, and quality of life (score SF-36) were compared between the two groups before and after treatment. The total effective rate of the OG was markedly higher than that of the CG. After treatment, the Barthel index, Fugl-Meyer assessment score, motor nerve conduction velocity, and sensory nerve conduction velocity and amplitude of the OG were obviously higher than those of the CG, and the SF-36 scores of the OG were higher than those of the CG in 8 dimensions. Rehabilitation training combined with Jiaji electroacupuncture intervention can dramatically promote the recovery of muscle group function and improve the quality of life of patients with upper limb peripheral nerve injury.
\end{abstract}

\section{Introduction}

Due to external trauma, upper limbs, including myelin sheath, axon, connective tissue, and peripheral nerve damage, are known as upper limb peripheral nerve injuries. Because of the compression of the upper limb nerve, ischemia, and hypoxia and the damage of other tissues around, it will affect the function of the upper limbs and lead to abnormal life and work [1-3]. However, the long-term side effects of upper limb nerve injuries and chronic neuropathic pains will influence the patients' psychological status and emotions, which greatly reduces their quality of life and treatment outcome $[4,5]$. At present, there are gradually reconstructive operations clinically, but a large number of patients lose the normal function of their upper limbs after the operation. Long-term nerve injury and inability to recover will also lead to lifelong disability of patients, so further postoperative recovery is needed to cure them [6-8].

After nerve reconstruction, the regeneration of motion axis takes a long time, and the recovery and healing cycle of peripheral nerve injury of upper limbs is slow, so long-term rehabilitation training is needed to prevent muscle atrophy and degenerative diseases and gradually activate the control ability of peripheral muscles, thus accelerating the recovery of injured function of patients [9, 10]. Jiaji electroacupuncture is an important means to treat nerve injury. Electric field can promote nerve regeneration and recovery of nerve conduction function and can alleviate the degree of muscle atrophy of patients [11]. Wang et al. [12] 
mentioned that Jiaji electroacupuncture intervention could promote the repair process of sciatic nerve injuries in rabbits. Li et al. [13] found that the intervention might reduce the spinal cord injuries of the acute spinal cord injured rats and significantly improve motor function by reducing the spinal cord effect of nerve cell apoptosis. After peripheral nerve injuries, it will gradually lead to apoptosis of corresponding neuron cell bodies, which may be due to the fact that nutrition conduction becomes abnormal after injuries, so the bodies cannot obtain neurotrophic factors and die [14]. Therefore, in many in vitro cell and different cell injury model studies, we found that glial cell-derived neurotrophic factor and brain-derived neurotrophic factor neurons grew outward, improved the peripheral nerve injury of animal models, and reduced apoptosis and autophagy $[15,16]$. Jing et al. [17] confirmed that electroacupuncture could significantly promote facial nerve regeneration by upregulating the expression of GDNF and N-cadherin in neurons, thereby inhibiting neuronal apoptosis and promoting the regeneration of peripheral facial nerve injury in rabbits. Thus, we observed the treatment plan of rehabilitation training combined with Jiaji electroacupuncture to see if it could promote treatment. The purpose of this study is to explore the effect of rehabilitation training combined with Jiaji electroacupuncture intervention on the recovery of upper limb peripheral nerve injury patients.

\section{Data and Methods}

2.1. Patient Data. A total of 114 patients with peripheral nerve injury of upper limbs in our hospital were collected from August 2017 to November 2019. Among them, 59 in the control group (CG) received rehabilitation training alone, including 37 males and 22 females, aged (38.2 \pm 8.7 ), while 65 in the observation group (OG) received rehabilitation training combined with Jiaji electroacupuncture intervention, including 45 males and 20 females, aged $(37.6 \pm 8.3)$. This study was consistent with the Declaration of Helsinki and was approved by the medical ethics committee of our hospital. All patients obtained and signed an informed consent form.

2.2. Inclusion and Exclusion Criteria. Inclusion criteria: all patients had peripheral nerve injuries and dysfunction of upper limbs, and they had undergone surgical nerve repair operation and intended to complete treatment and followup investigation.

Exclusion criteria were as follows: those who were complicated with other peripheral nerve diseases; those who could not tolerate the acupuncture electromyography test; those who complicated with rheumatoid arthritis type nervous system diseases; those who complicated with other bone injury diseases; and those who had cognitive dysfunction.

2.3. Treatment Methods. Patients in the CG received rehabilitation training, and the medical workers should assist them with joint activity and muscle strength training. Their upper limb ability and muscle strength were improved through specific treatment tasks, and they were trained to sensation. They were trained by sensory desensitization and reeducation, and joint loosening techniques were performed regularly. Those in the OG were treated with Jiaji electroacupuncture on the basis of the CG, with C6-T1 Jiaji points, 2 -inch filiform needles inclined to 1 -inch bilateral points, through connected pulse electrotherapy instrument, once a day, $30 \mathrm{~min} /$ time, for 8 weeks.

2.4. Nerve Conduction Assessment. The motor nerve conduction velocity (NCV) and $\mathrm{M}$ wave amplitude were evaluated by stimulating the median nerve and recording the corresponding motor response of the nerve. The elbow of the subject was completely extended, the recording electrode was directly located on the motor point of the myocardium, the reference one was put on the tendon of the intercondylar joint, and the ground was located on the palm. The median nerve was stimulated on the wrist between the flexor tendon of the hand and the medial side near the chest circumference of musculus biceps. The intensity was determined by increasing the amplitude gradually until the maximum $M$ wave was reached. Then, the amplitude was stimulated by $120 \%$ of this value during the test. Ten tests were carried out with a bandwidth of $10 \mathrm{~Hz}-1 \mathrm{kHz}$, and the amplitude of $M$ wave was also evaluated as an indication of the motor response strength. The scanning speed was $2 \mathrm{~s}$ per minute, and sensitivity is $2 \mu \mathrm{V}$ per minute. The stimulation duration and rate were $0.2 \mathrm{~s}$ and $3 \mathrm{~Hz}$, respectively.

2.5. Evaluation of Therapeutic Effects. Markedly effective: the pain symptoms basically disappeared, the nerve function basically returned to normal, and the nerve conduction velocity was remarkably improved; effective: the symptoms were alleviated, and the nerve function and conduction velocity were improved; ineffective: the symptoms were not alleviated, the nerve function and conduction velocity were not improved or even aggravated, and the total effective rate $=$ (markedly effective + effective) cases/total cases $\times 100 \%$.

2.6. Outcome Measures. The therapeutic efficacy between the two groups was counted and compared. Barthel index and Fugl-Meyer assessment score were compared before and after treatment; the former evaluated the patients' activity of daily living (ADL), 100 points in total, and the later assesses the patients' upper limb motor function, so as to evaluate the recovery of upper limb motor function, 66 points in total. Neuroelectrophysiological indexes of two groups motor nerve conduction velocity, sensory nerve conduction velocity, and amplitude were analyzed. The quality of life of patients in the two groups was observed by the SF-36 score, and the quality of life was evaluated from eight aspects: physiological function, social role, physical pain, health condition, energy, social and emotional function, and mental health, with a total score of 100 points for each item.

2.7. Statistical Methods. SPSS 20.0 (SPSS Co., Ltd., Chicago, the States) was used for statistical analysis. The continuous variables were expressed by the number of 
cases, average, and standard deviation. Both groups were compared by the independent $t$-test, the different periods between the same group were evaluated by the paired $t$ test, and the results were expressed by $t$. For classification variables, the data were expressed as the number or percentage of classification cases, detected by the chisquare test, and finally analyzed by the $X^{2}$ test. $P<0.05$ indicated that the difference was statistically marked.

\section{Results}

3.1. Clinical Data. By comparing the clinical data of patients in the two groups, we found that there was no remarkable difference in gender, age, time, site, and reason of injuries, nerve fracture degree, operation methods, and extent of damage between the two groups. It was comparable, as given in Table 1.

3.2. Therapeutic Effects. By comparing the therapeutic effects of the two groups, we found that there was no marked difference in the markedly effective and effective rates, and the total effective rate of the OG was markedly higher than that of the CG (Table 2).

3.3. ADL and Upper Limb Motor Function Changes. By comparing Barthel index and Fugl-Meyer assessment scores of two groups of patients, we found that two scores increased markedly, and the scores of the OG were obviously higher than those of the CG after treatment, as shown in Figure 1.

3.4. Neurophysiological Indicators. By comparing the neurophysiological indicators such as motor nerve conduction velocity, sensory nerve conduction velocity, and amplitude between the two groups, we found that the three after treatment were dramatically higher than those before treatment, and those of the OG were remarkably higher than those of the CG after treatment, as shown in Figure 2.

3.5. Quality of Life before and after Treatment. The patients' quality of life was evaluated based on the SF-36 scores of the two groups, and it was found that the scores of the OG in 8 dimensions were higher than those of the CG $(P<0.05)$, as given in Table 3.

\section{Discussion}

In this study, the therapeutic effects of the two groups were compared at first, and it was found that the total effective rate of the OG was markedly higher than that of the CG, which might indicate that the treatment scheme of rehabilitation training combined with Jiaji electroacupuncture is better than that of rehabilitation training alone. Then, we compared Barthel index and Fugl-Meyer assessment scores before and after admission. The former could better evaluate the patients' ADL, while the latter could effectively analyze upper limb disorders [18, 19]. We also discovered that the two scores of the two groups of patients increased significantly after treatment, which indicated that all of them improved their upper limb function and autonomous living function. Furthermore, we found that the scores of the OG were remarkably higher than those of the CG after treatment, which indicated that rehabilitation training combined with Jiaji acupuncture had a better recovery effect on the muscle group function of patients and could cure patients better.

Neurophysiological abnormalities can be detected by electromyography and nerve conduction research [20]. Hence, we detected electromyography indexes including motor nerve conduction velocity, sensory nerve conduction velocity, and amplitude before and after treatment in both groups. All the three in the OG were markedly higher than those in the CG after treatment, which also verified that rehabilitation training combined with Jiaji acupuncture therapy had better therapeutic advantages. He et al. [21] reported that electroacupuncture could ensure and improve the continuity between peripheral and central nerve, thus shortening the repair process of the damaged nerve, providing the basis for regeneration, and recovering motor conduction well. Many studies have mentioned that the treatment of peripheral nerve injury by electrical stimulation has a good therapeutic effect. For example, Yu et al. [22] found that electrical stimulation could enhance nerve regeneration and functional recovery, and the implementation of electrode point and 1/4 circle contact on the sciatic nerve resection model could significantly improve sciatic nerve function index, compound muscle action potential, and motor nerve conduction velocity scores and could better promote sciatic nerve regeneration and reduce muscle atrophy, with less mechanical damage to nerve trunk. Pan et al. [23] studied that electrical stimulation could remarkably treat peripheral neuropathy caused by diabetes, reduce the apoptosis of sciatic nerve cells in diabetic rats, and prevent sciatic nerve injury by inhibiting the occurrence of endoplasmic reticulum stress. At the end of the study, we counted and compared the quality of life of the two groups of patients. Because of severe neuropathic pain and the possibility of disability, the quality of life of those with peripheral nerve injury of upper limbs was not high [23]. Our research shows that the quality of life of patients with combined treatment was remarkably higher than that of those with rehabilitation training alone. 
TABle 1: Clinical data sheet.

\begin{tabular}{|c|c|c|c|c|}
\hline & Control group $(n=59)$ & Observation group $(n=65)$ & $X^{2} / t$ & $P$ \\
\hline \multicolumn{5}{|l|}{ Gender } \\
\hline Male & $37(62.71)$ & $45(69.23)$ & 0.587 & 0.444 \\
\hline Female & $22(37.29)$ & $20(30.77)$ & 0.695 & 0.393 \\
\hline Age (years) & $38.2 \pm 8.7$ & $37.6 \pm 8.3$ & & \\
\hline Injury time $(\mathrm{h})$ & $5.09 \pm 1.17$ & $5.04 \pm 1.19$ & 0.236 & 0.814 \\
\hline \multicolumn{5}{|l|}{ Injury site } \\
\hline Radial nerve & $24(40.68)$ & $29(44.62)$ & \multirow{3}{*}{0.874} & \multirow{3}{*}{0.646} \\
\hline Ulnar nerve & $17(28.81)$ & $27(41.54)$ & & \\
\hline Median nerve & $18(30.51)$ & $19(29.23)$ & & \\
\hline \multicolumn{5}{|l|}{ Cause of injuries } \\
\hline Traffic accidents & $16(27.12)$ & $13(20.00)$ & \multirow{4}{*}{3.917} & \multirow{4}{*}{0.271} \\
\hline Incised wound & $9(15.25)$ & $12(18.46)$ & & \\
\hline Hinge rolling injury & $20(33.90)$ & $31(47.69)$ & & \\
\hline Puncture wound & $14(23.73)$ & $9(13.85)$ & & \\
\hline \multicolumn{5}{|l|}{ Degree of nerve rupture } \\
\hline Incomplete fracture & $31(52.54)$ & $39(60.00)$ & \multirow[t]{2}{*}{0.700} & \multirow[t]{2}{*}{0.403} \\
\hline Complete fracture & $28(47.46)$ & $26(40.00)$ & & \\
\hline \multicolumn{5}{|l|}{ Operation modes } \\
\hline Repair of neurological defect & $34(57.63)$ & $40(61.54)$ & \multirow{3}{*}{0.282} & \multirow{3}{*}{0.869} \\
\hline Neuroanastomosis & $10(16.95)$ & $9(13.85)$ & & \\
\hline Neurolysis & $15(25.42)$ & $16(24.61)$ & & \\
\hline \multicolumn{5}{|l|}{ Extent of damage } \\
\hline Moderate injury & $25(42.37)$ & $22(33.85)$ & \multirow[t]{2}{*}{0.955} & \multirow[t]{2}{*}{0.328} \\
\hline Severe injury & $34(57.63)$ & $43(66.15)$ & & \\
\hline
\end{tabular}

TABLE 2: Therapeutic effect table.

\begin{tabular}{lcrr}
\hline & Control group $(n=59)$ & Observation group $(n=65)$ & \multicolumn{1}{c}{$X^{2}$} \\
\hline Markedly effective & $18(30.51)$ & $27(41.54)$ & 1.627 \\
Effective & $29(49.15)$ & $33(50.77)$ & 0.202 \\
Ineffective & $12(20.34)$ & $5(7.69)$ & 0.857 \\
Total effective rate & $47(79.66)$ & $60(92.31)$ & 4.181 \\
\hline
\end{tabular}

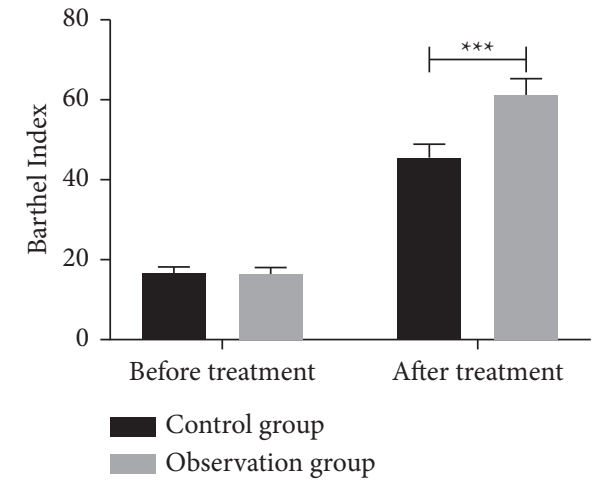

(a)

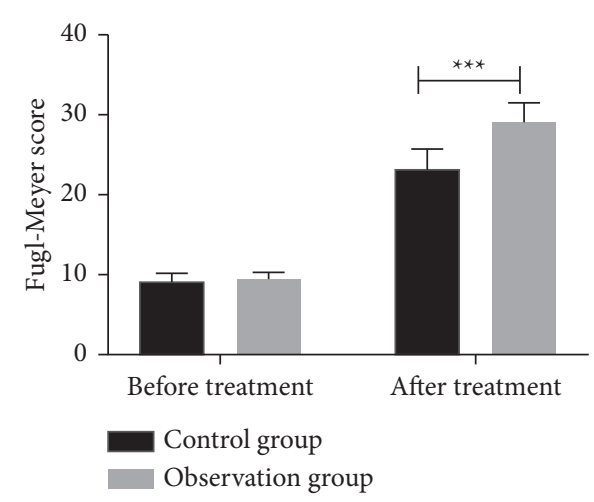

(b)

FIGURE 1: Changes of ADL and upper limb motor function. (a) The Barthel index of both groups after treatment is significantly higher than that before treatment and that of the observation group is significantly higher than that of the control group after treatment. (b) After treatment, the scores of Fugl-Meyer assessment scale in the two groups are significantly higher than those before treatment, and the observation group is significantly higher than that in the control group. 


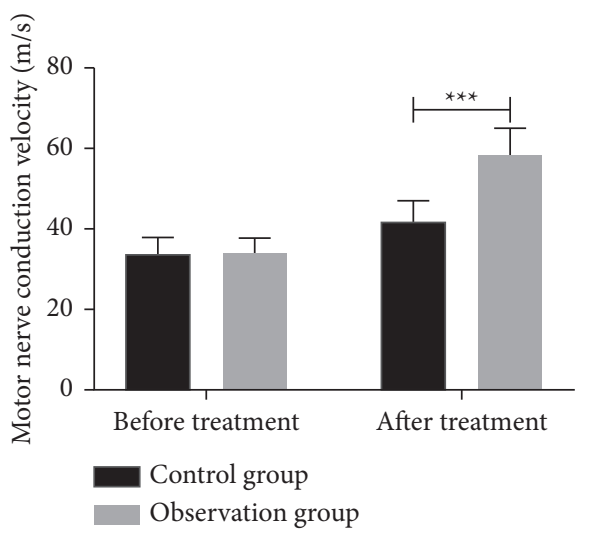

(a)

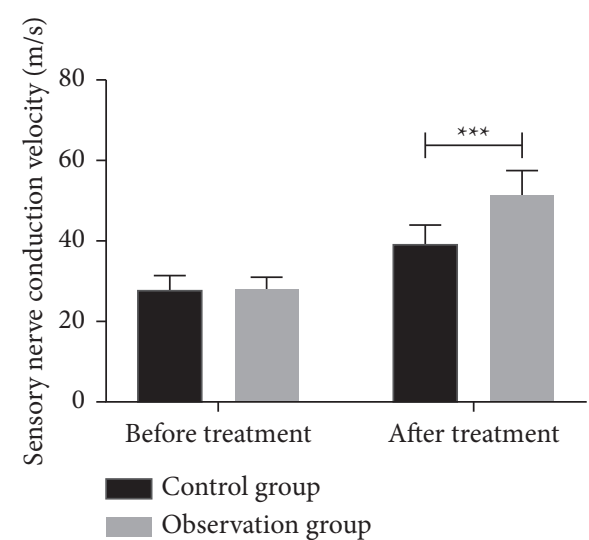

(b)

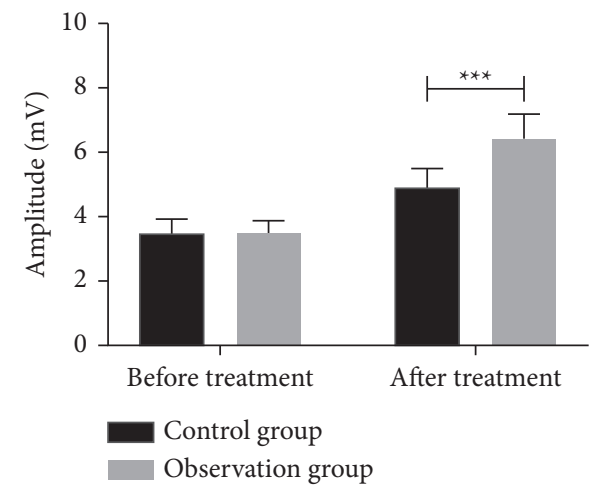

(c)

FiguRE 2: Changes of neurophysiological indicators before and after treatment. (a) Motor nerve conduction velocity of two groups of patients after treatment is significantly higher than that before treatment and that of the observation group is significantly higher than that of the control group after treatment. (b) Sensory nerve conduction velocity of the two groups after treatment is significantly higher than that before treatment, and the observation group is significantly higher than that of the control group after treatment. (c) After treatment, the amplitude of patients in the two groups is significantly higher than that before treatment and that in the observation group is significantly higher than that in the control group after treatment.

TABLE 3: SF-36 scoring results table.

\begin{tabular}{|c|c|c|c|c|}
\hline & Control group $(n=59)$ & Observation group $(n=65)$ & $X^{2}$ & $t$ \\
\hline Physiological function & $63.56 \pm 17.25$ & $70.26 \pm 16.93$ & 2.181 & 0.031 \\
\hline Mental health & $54.26 \pm 14.33$ & $66.52 \pm 16.23$ & 4.440 & $<0.001$ \\
\hline Somatic pain & $64.64 \pm 15.36$ & $74.84 \pm 12.75$ & 4.037 & $<0.001$ \\
\hline Emotional function & $60.62 \pm 15.38$ & $68.20 \pm 17.52$ & 2.549 & 0.012 \\
\hline Energy & $51.53 \pm 13.92$ & $57.42 \pm 13.55$ & 2.386 & 0.019 \\
\hline Social role & $51.37 \pm 22.15$ & $61.76 \pm 16.64$ & 2.970 & 0.004 \\
\hline Health status & $54.33 \pm 12.85$ & $62.39 \pm 11.24$ & 3.725 & $<0.001$ \\
\hline Social function & $52.46 \pm 13.65$ & $58.77 \pm 12.94$ & 2.642 & 0.009 \\
\hline
\end{tabular}

\section{Conclusion}

To sum up, rehabilitation training combined with Jiaji electroacupuncture can significantly promote the recovery of muscle group function and improve the quality of life of patients with upper limb peripheral nerve injury.

However, there are some shortcomings in our research. First of all, there are some differences in the surgical methods for patients, but the research on porcelain fracture has not explored this. Second, we have not further explored the specific mechanism of improvement, so we hope to carry out corresponding animal experiments to support our conclusions. At the moment, there are some new therapies for peripheral nerve reconstruction and recipient nerve regeneration, such as using allogeneic transplantation or other transplantation 
materials. It is also found that patients have better nerve regeneration after operation, the biological activity of cells has been improved, the inhibition of apoptosis has been suppressed, and the immune rejection is less [24], which may also become the focus of our future research.

\section{Data Availability}

The datasets used and/or analyzed during the current study are available from the corresponding author upon request.

\section{Conflicts of Interest}

The authors declare that they have no conflicts of interest.

\section{References}

[1] K. D. Bergmeister, L. Große-Hartlage, S. C. Daeschler et al., "Acute and long-term costs of 268 peripheral nerve injuries in the upper extremity," PLoS One, vol. 15, no. 4, Article ID e0229530, 2020.

[2] J. Isaacs and A. R. Cochran, "Nerve transfers for peripheral nerve injury in the upper limb," The Bone \& Joint Journal, vol. 101-B, no. 2, pp. 124-131, 2019.

[3] K. Sluys Prignitz, S. Justine, and S. Richmond Therese, "Health related quality of life and return to work after minor extremity injuries: a longitudinal study comparing upper versus lower extremity injuries," ITS Journal - Intelligent Transportation Systems Journal, vol. 47, pp. 824-831, 2016.

[4] Z. Peixun, H. Na, Y. Kou, Y. Xiaofeng, and B. Jiang, "Peripheral nerve intersectional repair by bi-directional induction and systematic remodelling: biodegradable conduit tubulization from basic research to clinical application," Artificial cells, nanomedicine, and biotechnology, vol. 45, pp. 1464-1466, 2017.

[5] O. Heary Kartemus, A. W. K. Wong, C. L. Lau Stephen, J. Denglar, M. R. Thompson, and L. W. Crock, C. B. Novak, B. A. Philip, S. E. Mackinon, Quality of life and psychosocial factors as predictors of pain relief following nerve surgery," vol. 19, Article ID 1558944720911213, 2020.

[6] E. Magistroni, G. Parodi, F. Battiston, and L. B. Dahlin, "Cold intolerance and neuropathic pain after peripheral nerve injury in upper extremity," Journal of the Peripheral Nervous System: JPNS, vol. 25, pp. 184-190, 2020.

[7] C. N. P. Bruyns, J.-B. Jaquet, T. A. R. Schreuders, S. Kalmijn, P. D. L. Kuypers, and S. E. R. Hovius, "Predictors for return to work in patients with median and ulnar nerve injuries," The Journal of Hand Surgery, vol. 28, no. 1, pp. 28-34, 2003.

[8] H. E. Rosberg, K. S. Carlsson, S. Höjgård, B Lindgren, G Lundborg, and L. B Dahlin, "Injury to the human median and ulnar nerves in the forearm--analysis of costs for treatment and rehabilitation of 69 patients in southern Sweden," The Journal of Hand Surgery, vol. 30, no. 3, pp. 35-39, 2005.

[9] S. Agnes, A. Hruby Laura, P. Cosima, A. M. Johannes, and C. A. Osker, "Rehabilitation of upper extremity nerve injuries using surface EMG biofeedback: protocols for clinical application," Front Neruscience, vol. 12, p. 906, 2018.

[10] A. S. Pagnussat, S. M. Michaelsen, M. Achaval et al., "Effect of skilled and unskilled training on nerve regeneration and functional recovery," Brazilian Journal of Medical and Biological Research, vol. 45, no. 8, pp. 753-762, 2012.

[11] Y. Fan and Y. Wu, "Effect of electroacupuncture on muscle state and infrared thermogram changes in patients with acute lumbar muscle sprain," Journal of traditional Chinese medicine $=$ Chung $i$ tsa chih ying wen pan, vol. 35, pp. 499-506, 2015.

[12] Y. Wang, C.-F. Dong, R.-N. Xu, Z.-N. Guo, L.-L. Zheng, and Y.-M. Yuan, "Effect of electroacupuncture at "Jiaji" (EX-B 2) points combined with nerve mobilization on motor function and mRNA and protein expression of RhoA in rabbits with sciatic nerve injury," ITS Journal - Intelligent Transportation Systems Journal, vol. 39, pp. 625-631, 2019.

[13] X.-N. Li, L. Wu, C. Lei, Z.-W. Qi, J. -L. Mei, and N. Li, “[Effect of electroacupuncture intervention at different time-points on locomotor function and apoptosis in rats with acute spinal cord injury]," ITS Journal - Intelligent Transportation Systems Journal, vol. 41, pp. 492-496, 2016.

[14] X. Wang, W. Ma, T. Wang et al., "BDNF-TrkB and proBDNFp75NTR/Sortilin signaling pathways are involved in mitochondria-mediated neuronal apoptosis in dorsal root ganglia after sciatic nerve transection," CNS \& Neurological Disorders - Drug Targets, vol. 19, no. 1, pp. 66-82, 2020.

[15] A. K. Chou, M. C. Yang, H. P. Tsai et al., "Adenoviral-mediated glial cell line-derived neurotrophic factor gene transfer has a protective effect on sciatic nerve following constriction-induced spinal cord injury," PLoS One, vol. 9, Article ID e92264, 2014.

[16] P. J. Kingham, M. K. Kolar, L. N. Novikova, L. N. Novikov, and M. Wiberg, "Stimulating the neurotrophic and angiogenic properties of human adipose-derived stem cells enhances nerve repair," Stem Cells and Development, vol. 23, no. 7, pp. 741-754, 2014.

[17] F. Jing, L. Gao, H.-H. Li, Q.-L. Yuan, and L.-J. Li, “Electroacupuncture promotes peripheral nerve regeneration after facial nerve crush injury and upregulates the expression of glial cell-derived neurotrophic factor," Neural Regen Res, vol. 14, pp. 673-682, 2019.

[18] J. Wang, L. Tian, Z. Zhang et al., "Scalp-acupuncture for patients with hemiplegic paralysis of acute ischaemic stroke: a randomized controlled clinical trial," Journal of Traditional Chinese Medicine, vol. 40, pp. 845-854, 2020.

[19] A. Martino Cinnera, S. Bonnì, M. C. Pellicciari et al., "Health-related quality of life (HRQoL) after stroke: p," Topics in Stroke Rehabilitation, vol. 27, no. 7, pp. 534-540, 2020.

[20] G. Andreisek, D. W. Crook, D. Marincek, and D. Weishaupt, "Peripheral neuropathies of the median, radial, and ulnar nerves: MR imaging features," RadioGraphics: A Review Publication of the Radiological Society of North America, Inc, vol. 26, pp. 1267-1287, 2006.

[21] G.-H. He, R. Jing-Wen, Y.-S. Zeng, X. Zhou, Y. Ding, and G.-H. Zhou, "Improvement in acupoint selection for acupuncture of nerves surrounding the injury site: electro-acupuncture with Governor vessel with local meridian acupoints," Neural Regen Res, vol. 10, pp. 128-135, 2015.

[22] A.-P. Yu, Y.-J. Shen, Y.-Q. Qiu et al., "Comparative effects of implanted electrodes with differing contact patterns on peripheral nerve regeneration and functional recovery," $\mathrm{Neu}$ roscience Research, vol. 145, pp. 22-29, 2019.

[23] H. Pan, H. Huang, L. Zhang, S. Ma, H. Yang, and H. Wang, “"Adjusting internal organs and dredging channel" electroacupuncture treatment prevents the development of diabetic peripheral neuropathy by downregulating glucose-related protein 78 (GRP78) and caspase-12 in streptozotocin-diabetic rats," Journal of Diabetes, vol. 11, no. 12, pp. 928-937, 2019.

[24] Z. Li, S. Zhang, J. Li, H. Zeng, Y. Wang, and Y. Huang, "Nerve regeneration in rat peripheral nerve allografts: e," Journal of Comparative Neurology, vol. 527, no. 17, pp. 2885-2895, 2019. 